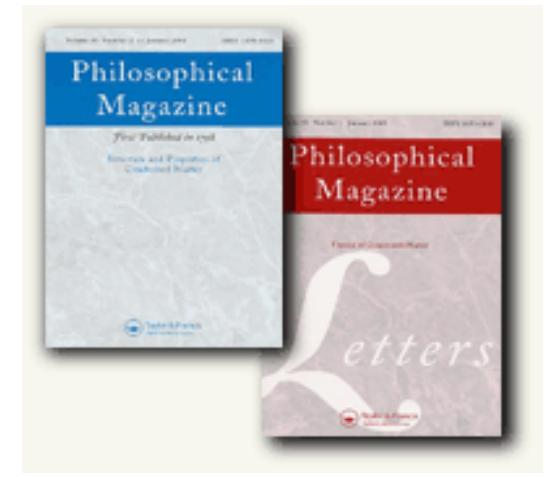

\title{
Evolution of mechanical response and dislocation microstructures in small scale specimens under slightly different loading conditions
}

\begin{tabular}{|r|l|}
\hline Journal: & Philosophical Magazine \& Philosophical Magazine Letters \\
\hline Manuscript ID: & TPHM-09-Jun-0263.R1 \\
\hline Journal Selection: & Philosophical Magazine \\
\hline Author: & 24-Jul-2009 \\
\hline & $\begin{array}{l}\text { Complete List of Authors: } \\
\text { Sotz, Christian; Erich Schmid Institut, Austrian Academy of } \\
\text { Gumbsch, Peter; IZBS, Universität Karlsruhe (TH); IWM, } \\
\text { Fraunhofer Institut für Werkstoffmechanik } \\
\text { Kraft, Oliver; IZBS, Universität Karlsruhe (TH); Institut für } \\
\text { Materialforschung II, Forschungszentrum Karlsruhe }\end{array}$ \\
\hline Keywords: & dislocation dynamics, dislocation structures, simulation \\
\hline Keywords (user supplied): & micro samples, boundary conditions \\
\hline \hline &
\end{tabular}

\section{ScholaroNE}




\title{
Evolution of mechanical response and dislocation microstructures in small scale specimens under slightly different loading conditions
}

\author{
Jochen Senger $^{1 *}$, Daniel Weygand ${ }^{1}$, Christian Motz ${ }^{1,2}$, Peter Gumbsch ${ }^{1,3}$, \\ and Oliver Kraft ${ }^{1,4}$
}

${ }^{1}$ IZBS, Universität Karlsruhe (TH), Kaiserstr. 12, 76131 Karlsruhe, Germany

${ }^{2}$ Erich Schmid Institute, Austrian Academy of Sciences, Jahnstr. 12, 8700 Leoben, Austria

${ }^{3}$ IWM, Fraunhofer Institut für Werkstoffmechanik, Wöhlerstr. 11, 79108 Freiburg, Germany

${ }^{4}$ Institut für Materialforschung II (IMF II), Forschungszentrum Karlsruhe, Germany

* Corresponding author

jochen.senger@kit.edu

Phone: +49 (0)721 608-8509

Fax: +49 (0)721 608-4364

\begin{abstract}
In small dimensions, the flow stress of metallic samples shows a size-dependence such that smaller is stronger, even in nominally strain gradient-free loading conditions. However, the role of the boundary conditions in miniaturized tension or compression tests on the mechanical response and dislocation structure has not been studied in detail. In simulations performed with a 3-D discrete dislocation dynamics tool, initial, welldefined dislocation microstructures are loaded in tension with different boundary conditions including superimposed torsion moments. The influence of the loading conditions on details of the evolving dislocation microstructure is investigated by using identical starting configuration. An additional torsion moment significantly influences the dislocation activity since forest-dislocations are generated, but size effect of the flow stress is found to be unchanged.
\end{abstract}

Keywords: dislocation structures, micro samples, simulation, boundary conditions, dislocation dynamics

\section{Introduction}

In the last few years, compression [1-7] and tension experiments [8] on micron- and submicron-scale metallic samples have drawn quite some attention. It was found in these experiments that the flow stress of the tested metallic specimens is sizedependent. Flow stress increases with decreasing sample size. From a continuum mechanical point of view this is somewhat surprising since the loading condition is nominally uniaxial and free of imposed strain gradients. Therefore, even advanced continuum descriptions like strain gradient plasticity can not capture the phenomenon. Details of the dislocation behaviour must be considered to explain the phenomenon. 
Several qualitative explanations have been put forward, including dislocation starvation [5, 7] or truncation of dislocation sources [9]. Furthermore, discrete dislocation dynamics (DDD) simulations schemes have been applied to study these mechanisms in more detail $[10,11,12]$. Parthasarathy et al. [10] confirmed that higher flow stresses can be attributed to smaller source lengths in smaller samples. Similarly, Senger et al. [11] found that dislocation reactions take place in larger samples such that larger and therefore weaker dislocation sources are formed through dislocation reactions. This trend has been also observed by Motz et al. [12] for relaxed, initially pinning point free dislocation structures.

Apart from these mechanistic explanations, other aspects concerning the experimental set-up and the impact of focused ion beam (FIB) damage on the size effect are still under debate [13-15]. The central question in this debate is how the flow stress and the evolution of the dislocation microstructure depend on experimental aspects like misalignment leading to non-uniform loading or constraints imposed by the boundary conditions [16].

To address misalignment effects, DDD simulations for uniaxial loading of micrometre-sized samples with superimposed torsion were performed in this study. Such torsion moments can occur in experiments due to alignment imperfections between sample and loading gadget [8] and is, of course, highly undesired but typically beyond control for experiments at the micrometer scale.

\section{Simulation method}

Simulations of deformation experiments on small-scale columns are performed with the 3-dimensional discrete dislocation dynamics simulation tool described in $[17,18]$. Apart from the variations in the loading conditions, the simulations reported here are similar to those in [11]. Briefly, the crystallographic orientation is taken from the 
experiments of [8]. The tensile axis of the columns is $<\overline{2} 34>$ where single slip is expected. One side of the pillar is perpendicular to the $<0 \overline{4} 3>$ direction. The initial dislocation structure consists of Frank-Read sources of random orientation with a uniform length of $220 \mathrm{~nm}$. These sources are equally distributed on the 12 glide systems such that an initial dislocation density of $2.1 \times 10^{13} \mathrm{~m}^{-2}$ is achieved for all column sizes. The columns have a square cross-section and given column sizes reflect the length of the edge of the square. All initial Frank-Read sources are placed within a cylinder around the tensile axis with a diameter equal to the square size. However, the presented dislocation densities are related to the complete volume. The investigated column sizes are between 0.5 and $2.0 \mu \mathrm{m}$ with a fixed aspect ratio of 1:3. Eight simulations with random position of the initial Frank-Read sources are performed for specimens with a diameter of $0.5 \mu \mathrm{m}$ and five simulations for $1 \mu \mathrm{m}$ samples for each condition. Only two simulations are performed for the largest columns, due to the high computational costs. This can be justified since statistical variations are smaller in the larger sample as shown in [11] where for exactly this sample size the yield strength and the stresses in the plastic regime were within a few MPa for different initial configurations.

Columns with identical initial dislocation structures are loaded under three different boundary conditions. In all simulations, the displacement of the bottom of the column is fixed. Tensile loading along the long column axis is the main loading component, on which torsion as a secondary component is superimposed. Within the small strain assumption of our DDD model, tensile and compression simulations are statistically equivalent. 
For the first loading condition, pure tension (PT), the displacement at top surface of the column, is prescribed in the tensile direction only and the in-plane displacements are free allowing sideward motion. This corresponds to no friction between a pillar and the flat punch in a compression test or to an experimental setup, which is laterally very compliant both in tension or compression.

For the second boundary condition, constrained tension (CT), the additional constrain is that the in-plane displacements at the top surface are fixed during the entire test. This condition corresponds to tests with a laterally stiff experimental setup where both ends of the sample are clamped in tension or high friction in compression.

In the third boundary condition, the tension-torsion test (TT), a torsion moment is superimposed and the column top displacement is laterally fixed. The torsion is generated by a rotation of the top surface around the tensile axis. This loading condition is meant to mimic a non-ideal situation due to initial misalignment within the experimental setup. Of course, other superimposed constraints can be imagined, e.g. additional bending [19]. Table 1 summarizes the imposed constraints.

All simulations are performed with displacement control with a constant strain rate $\dot{\varepsilon}$ of $5000 \mathrm{~s}^{-1}$. Variation of the strain rate between $100 \mathrm{~s}^{-1}$ and $5000 \mathrm{~s}^{-1}$ in PT-tests shows no significant change in flow stress level and size effect. Therefore, we argue that strain rate effects do not play a significant role in our simulations. In the simulations, it is assumed that the linearly increasing torsion occurs only during the initial loading. This is meant to reflect the self-alignment of the column or tensile sample with respect to the loading device in a real experiment. For this, the torsional displacements components are superimposed and continuously increased until the total tensile strain reaches $0.1 \%$. This tensile strain corresponds to a tensile stress of about $72 \mathrm{MPa}$ which is just below the activation stress of the initial Frank-Read 
sources. Two torsion rates are chosen so that torque angles of $\varphi=0.5^{\circ}$ and $2.0^{\circ}$ are reached at $0.1 \%$ tensile strain. This corresponds to shear stresses caused by the torsion angle of 40 and $160 \mathrm{MPa}$, respectively, at the surface of a cylinder around the tensile axis with a diameter equal to the square size. These values are independent of the sample size because the shear stresses depend only on the shear angle according to Hooke's law. Then, for reaching tensile strains larger than $0.1 \%$, the torsion angle reached at $0.1 \%$ tensile strain is kept constant and only the tensile strain is increased further.

In the subsequent part, yield strength $\mathrm{R}_{\mathrm{p} 0.01 \%}$ is measured at $0.01 \%$ plastic tensile strain and the discussed flow stress $\mathrm{Rp} 0.2 \% \mathrm{R}_{\mathrm{p} 0.2 \%}$ are taken at $0.2 \%$ plastic tensile strain.

\section{Results}

\subsection{Samples with $0.5 \mu \mathrm{m}$ diameter}

The averaged stress-strain curves of the simulations with eight different initial dislocation configurations and the corresponding evolution of the dislocation density in $0.5 \mu \mathrm{m}$ samples for different loading conditions are shown in Fig. 1. Eight identical initial structures have been used in each case to illustrate the effect of the different boundary conditions.

In pure tension tests, the periodic activation of one single source is usually observed. This leads to plastic deformation without strain hardening. The flow stress is constant and no overall increase in dislocation density is observed.

In contrast to pure tension, individual configurations show either a constant flow stress or hardening behaviour under CT-conditions. Dislocations pile up at the top and bottom surface and dislocation density increases continuously. With a superimposed torsion angle, only flow stress is similar to both tensile tests. In the TT- 
test, the average yield strength is reduced with an increased torsion angle. Superimposed torsion generally leads to larger dislocation densities as in CT-tests. In the $2^{\circ}$ twisted samples, dislocation density increases already during the deformation to $0.1 \%$ tensile strain thereafter the torsion rate is set equal to zero but dislocation density still increases significantly.

Fig. 2 shows superimposed snapshots of the simulated identical dislocation structure at various time intervals of the simulation under different boundary conditions. Fig. 2a shows that active slip occurs mostly on one plane (blue plane marked by an arrow). With the CT-boundary condition and the very same initial dislocation structure, the same glide plane is active but more dislocation activity on a parallel glide plane as well as on other glide systems is observed (Fig. 2b).

In the simulation with an additional twist to a torsion angle of $0.5^{\circ}$ using boundary condition TT, it is clearly seen that beside the marked slip plane more slip systems were activated compared to the PT- and CT-conditions. In this particular simulation, the yield strength at $0.01 \%$ is not reduced compared to the tension tests. However, the flow stress for this configuration is reduced compared to the PT case for small plastic strains. This can be attributed to the multiaxial stress state that increases the activation of more slip systems as confirmed by the observed dislocation structure shown in Fig. 2c.

The superimposed structure of the $2.0^{\circ}$ twisted sample is split in the part where both, torsion angle and tensile displacement, are linearly increased (Fig. 2d) and in the part where the torsion angle is constant (Fig. 2e). Long dislocation segments (light blue) are formed in the beginning along the torsion axis which cannot easily leave the specimen due to the torsion component of the stress field (Fig. 2d). This is in contrast to dislocations activated on other slip systems where they can leave 
the sample through the free surfaces and, thus, not lead to an increase of the dislocation density. At higher tensile strains, activity starts on glide planes which are preferred under tension. The dislocations which are activated by the torsion are still in the sample and can act as obstacles. The dislocation microstructure at $0.8 \%$ tensile strain is presented in Fig. $2 \mathrm{f}$. With the twist angle of $2.0^{\circ}$, a reduced activity of the most active source in the other simulations (marked by the arrow in Fig. 2a-c) is observed. Instead, several other sources on different glide planes are activated. They produce more cross-slip events. The cross-slipped dislocation often leaves such segments along the specimen axis as shown in Fig. $2 \mathrm{f}$.

In the presented sample, the flow stress in the TT-test $\left(2.0^{\circ}\right)$ is slightly raised with work-hardening for tensile strains larger than $0.65 \%$ compared to the PT-test since dislocation on less preferred glide systems have to be activated. In other simulations, where flow stresses in PT- and TT-test are similar, the same source was found to be active under both conditions.

\subsection{Samples with $1.0 \mu \mathrm{m}$ diameter}

For samples with side length of $1.0 \mu \mathrm{m}$, simulations with the same boundary conditions as above were conducted in order to assess whether the size effect on plasticity [11] is influenced by the boundary conditions. For both the PT- and CTboundary conditions (Fig. 3) a rather similar averaged overall behaviour without any appreciable work-hardening is observed. The yield drop can be explained with the generation of new dislocation sources which are longer than the initial Frank-Read sources as reported in [11]. Increasing sample diameter results in a reduced variation of flow stress for all boundary conditions confirming previous results [11]. In the strain range from $0.4-0.8 \%$, the spread of flow stress is on a similar level within each boundary condition. The individual stress-strain curves of the samples with 
superimposed torsion angle are found to be systematically below the PT- and CTcurve at low strains, reach or exceed the stresses of the PT- and CT-curves for larger strains. The average flow stress of the $2.0^{\circ}$ twisted samples reaches the flow stress of both tension tests only for strains larger than $0.35 \%$. Here, yield strength $\mathrm{R}_{\mathrm{p} 0.01 \%}$ is systematically reduced in specimens with superimposed torsion. The scatter of the flow stress in $1.0 \mu \mathrm{m}$ specimens is less pronounced than in the $0.5 \mu \mathrm{m}$ samples for all tested boundary conditions.

The dislocation density is increasing more strongly for the CT-tests compared to PT-tests. In both cases, several sources on parallel glide planes are active at the same time (see example of dislocation structure in Fig. 4), but for PT-tests, glide is concentrated on fewer planes. In the CT-condition, there are sources active on planes which cut the top or bottom surface of the specimen and dislocations pile-up occur on these planes. This causes a major contribution to the increase in dislocation density which is observed in these simulations. However, for the used aspect ratio of 1:3, dislocation sources away from the constrained ends of the sample continue to be active and, thus, hardening does not occur. In the TT-test with the angle of $0.5^{\circ}$, activity on planes with different glide plane orientations compared to the active planes in PT- and CT-tests occurs. However, activity on these secondary planes is low compared to the primary planes (Fig. 4c).

For twisting to an angle of $2^{\circ}$, a stronger activity on all four glide planes is observed. Even at small strain of $0.1 \%$, the dislocation density has increased by about a factor of 2.5 from the initial value (superimposed structure from 0 to $0.1 \%$ total strain in Fig. 4d). Again, long dislocation segments (light blue) are formed by strain gradients in the initial plastic regime. With increasing tensile strains, sources on glide planes with a high Schmid factor are activated. In Fig. 4e parallel planes (blue) can 
been seen which are mainly generated after $0.1 \%$ tensile strain. Fig $4 \mathrm{f}$ highlights the complexity of the final microstructure. In the samples presented in Fig. 4, flow stress in the $2.0^{\circ}$ twisted sample is lower compared to PT-tests for tensile strains smaller than $0.6 \%$.

\subsection{Samples with $2.0 \mu \mathrm{m}$ diameter}

In $2.0 \mu \mathrm{m}$ samples, yield strength is further decreased in TT-tests (Fig. 3). As expected, averaged flow stresses are lower than in $1.0 \mu \mathrm{m}$. For this diameter, extreme softening behaviour in PT- and CT-tests is related to short initial sources compared to the sample diameter. In plastic regime, longer, weaker sources are generated [11]. Again, superimposed torsion results in a higher dislocation density.

\section{Discussion}

The aim of this study is to elucidate the influence of the boundary conditions on the dislocation behaviour in micrometre-sized samples during tensile loading. This is important because in experiments, pile-ups can occur, e.g. by hindered dislocation escape due to the indenter [3] or by low aspect ratios [20]. We have found that for tensile loading a constraint of the lateral deformation at the top and bottom of the specimen does not significantly affect the stress-strain behaviour for the deformation that can be reached in our simulations. If the displacement of the top surface perpendicular to the tensile axis is prohibited (like in CT-tests) in a $0.5 \mu \mathrm{m}$ specimen, slip on several parallel planes of the primary slip system as well as on planes with lower Schmid factors, occurs. In $1.0 \mu \mathrm{m}$ specimens more sources on parallel glide planes are activated in the CT-condition compared to PT. The concentration of slip on distinct glide planes has also been observed in experiments [8]. Some of these additional glide planes cut the top or bottom surface resulting in dislocation pile-ups. Due to the aspect ratio of 1:3, however, glide planes in the middle of the sample 
remain active and, thus, the constraint effect is minimized in agreement to experimental observations [3]. Also, no work-hardening is observed in the presented PT- and CT-tests.

In TT-condition, the yield strength, determined from the tensile load, decreases for all sample sizes with increasing torsion angle. While in small samples, yield strength scatters and can also be equal to tension tests, the onset of plasticity in large samples is always reduced without a variation of yield strength. Furthermore, only in the thin specimens flow stress follows a near-elastic range when the first, temporary active source is shut-down in a zone of low stress. For tension and torsion, contrary to tensile loading, the resolved shear stress on a dislocation source depends on the exact source position and the angle to the torsion axis [21]. The maximum shear stress depends only on the torsion angle, but due to the constant aspect ratio not on the specimen size. This leads to two zones on each glide system where the resolved shear stress caused by the torsion is equal to zero. In large samples with many sources, the probability is large for an initial Frank-Read source to be near the surface in a zone of high stress. Therefore, in contrast to the small ones, no scatter in yield strength is observed for these samples. The initial FR sources are distributed over the entire cross-section. For large samples there is a probability close to one to find a source in the highly stressed zone, leading to a small scatter in the yield strength which is not the case in small samples. Here yield stresses similar to the PT and CT loading conditions are also observed.

An important influence of the boundary conditions can be seen in the evolution of the dislocation density. In $0.5 \mu \mathrm{m}$ specimens under tensile loading, dislocation density is at a constant level, apart from the temporary increases due to source activation, as dislocation can leave the sample without multiplication $[5,7]$. 
Due to strain gradients introduced by a superimposed small torsion angle, the dislocation density increases significantly (Fig. 1). The larger the torsion angle, the stronger the increase of the dislocation density. This is in agreement with the argument that geometrically necessary dislocations are formed as a function of strain gradients [22]. According to [22], the dislocation density is calculated which is necessary to compensate the plastic torsion angle reached at $0.1 \%$ tensile strain. The calculated values are found to be lower than the measured one. The difference increases with increasing torsion rate and sample size. However, due to the chosen crystallographic orientation, the formed long dislocations (light blue) have no pure screw character and reside on an inclined glide plane with respect to the torsion axis. Furthermore, dislocation reactions in larger samples contribute to the higher density. Due to the torsion, source activation may occur on other glide systems than without torsion since the resolved shear stresses become a strong function of position in the sample. This leads to multi-slip behaviour and the observed occurrence of long dislocation lines almost parallel to the sample axis. These long dislocation segments (light blue lines in Fig. 2d-ef and 4d-ef) occur due to source activation in the sector with a high resolved shear stress generated by the imposed torsion angle. When these dislocations reach a zone with a small shear stress near the centre or the sector with low resolved shear stresses, they stop and may act as obstacles for gliding dislocations on other slip systems. Further motion is only possible by the long range interaction with other dislocations. In the investigated strain range, however, the flow stress is not significantly affected by these forest dislocations as active sources are hardly pinned especially in the highly stress regions near to the surface. Hardening due to the increase in dislocation density in tension tests with superimposed torsion is less pronounced as in bending tests [19]. 
Fig. 5 summarizes the mean values of flow stress $R_{p 0.2 \%}$ at $0.2 \%$ plastic strain for all simulations. The size effect that has been reported before [11] is not significantly influenced by the loading condition even if the dislocation microstructure and densities are quite different. This is a quite surprising observation since strain gradient effects as present in the twisted samples were expected to have a more pronounced effect. Compared to both tensile boundary conditions, however, the superimposed torsion angle of $2.0^{\circ}$ leads only to an increase of the standard deviation. to some extent. This may be attributed to the inhomegeneous stress state and source statisties in small samples.

\section{Conclusion}

In the presented simulations, it is found that the evolution of the same initial dislocation microstructure is strongly affected by the boundary conditions. Yield strength $\mathrm{R}_{\mathrm{p} 0.01 \%}$ is reduced with superimposed torsion because of higher shear stresses at low tensile strains. A transition from single to multiple slip with the occurrence of forest dislocations in the twisted samples has been observed. However, the flow stress $\mathrm{R}_{\mathrm{p} 0.2 \%}$ in the investigated strain regime and the influence of sample size are not significantly affected by the boundary constraints and the resulting forest dislocations.

Acknowledgement

The financial support of the European Commission NANOMESO Project under contract number NMP3-CT-2006-016710 and Deutsche Forschungsgemeinschaft (DFG) under project Gu367/18-2 is gratefully acknowledged. 


\section{References}

[1] M. D. Uchic, D. M. Dimiduk, J. N. Florando and W. D. Nix, Science 305 (2004) p.986.

[2] M. D. Uchic and D. M. Dimiduk, Mater. Sci. Eng. A 400-401 (2005) p.268.

[3] D. Kiener, C. Motz and G. Dehm, J. Mater. Sci. 43 (2008) p.2503.

[4] C. A. Volkert and E. T. Lilleodden, Phil. Mag. 86 (2006) p.5567.

[5] W. D. Nix, J. R. Greer, G. Feng and E. T. Lilleodden, Thin Solid Films 515 (2007) p.3152.

[6] K. S. Ng and A. H. W. Ngan, Acta Mater. 56 (2008) p.1712.

[7] J. R. Greer and W. D. Nix, Phys. Rev. B 73 (2006) p.245410.

[8] D. Kiener, W. Grosinger, G. Dehm and R. Pippan, Acta Mater. 56 (2008) p.580.

[9] D. M. Dimiduk, M. D. Uchic, S. I. Rao, C. Woodward and T. A. Parthasarathy, Model. Simul. Mater. Sci. Eng. 15 (2007) p.135.

[10] T. A. Parthasarathy, S. I. Rao, D. M. Dimiduk, M. D. Uchic and D. R. Trink, Scripta Mater. 56 (2007) p.313.

[11] J. Senger, D. Weygand, P. Gumbsch and O. Kraft, Scripta Mater. 58 (2008) p.587.

[12] C. Motz, D. Weygand, J. Senger and P. Gumbsch, Acta Mater. 57 (2009) p. 1744 .

[13] D. Kiener, C. Motz, M. Rester, M. Jenko and G. Dehm. Mater. Sci. Eng. A 459 (2007) p.262.

[14] S. Shim, H. Bei, M. K. Miller, G. M. Pharr and E. P. George, Acta Mater. 57 (2009) p.503.

[15] R. Maaß, S. van Petegem, D. Grolimund, H. van Swygenhoven, D. Kiener and G. Dehm, Appl. Phys. Lett. 92 (2008) p.071905.

[16] D. Raabe, D. Ma and F. Roters, Acta Mater. 55 (2007) p.4567.

[17] D. Weygand, L. H. Friedman, E. van der Giessen and A. Needleman, Model. Simul. Mater. Sci. Eng. 10 (2002) p.437.

[18] D. Weygand and P. Gumbsch, Mater. Sci. Eng. A 400-401 (2005) p.158.

[19] C. Motz, D. Weygand, J. Senger and P. Gumbsch, Acta Mater. 56 (2008) p.1942.

[20] D. Kiener, W. Grosinger and G. Dehm, Scripta Mater. 60 (2009) p.148.

[21] J. P. Hirth and J. Lothe. Theory of Dislocations, McGraw-Hill, 1968.

[22] N. A. Fleck, G. M. Muller, M. F. Ashby and J. W. Hutchinson, Acta Metall. 42 (1994) p.475. 
Figure 2: Superimposed dislocation structure in a $0.5 \mu \mathrm{m}$ specimen: (a) pure tension, (b) constrained tension, (c) tension and torsion with a torsion angle of $0.5^{\circ}$. Sample in (d) shows the superimposed structure in the tensile strain range from $0-0.1 \%$ where torsion angle and tensile strain are linearly increased (TT: $\left.2.0^{\circ}\right)$. In (e), the superimposed structure from tensile strain $0.1 \%$ up to $0.8 \%$ is presented where only tensile strain is increased. The dislocation structure of the sample loaded with TT $\left(2.0^{\circ}\right)$ at $0.8 \%$ tensile strain is shown in (f). Active glide plane under tension and $0.5^{\circ}$ torsion is marked with a black arrow.

Figure 3: Averaged stress-strain curves (thick lines) and evolution of dislocation density (thin lines) in $1.0 \mu \mathrm{m}$ thin specimens and $2.0 \mu \mathrm{m}$ samples (dashed lines) under different loading conditions. Error bars indicate the standard deviation of the flow stresses in the $1.0 \mu \mathrm{m}$ samples at 0.1 and $0.5 \%$ tensile strain.

Figure 4: Superimposed dislocation microstructure in a $1.0 \mu \mathrm{m}$ sample: (a) pure tension, (b) constrained tension (CT), (c) tension and torsion (TT) with a torsion angle of $0.5^{\circ}$. The microstructure of the $2.0^{\circ}$ twisted sample is decomposed in the tensile strain (d) from $0-0.1 \%$ and (e) for strains from $0.1-0.8 \%$. Current dislocation structure of the TT $\left(2.0^{\circ}\right)$ sample at $0.8 \%$ tensile strain is illustrated in (f).

Figure 5: Size effect in micron samples. Mean values of flow stresses at $0.2 \%$ plastic strain are given by the symbols. Error bars indicate the standard deviation. Two straight lines described by a power-law are given. For clarity, stress values are presented abreast. 


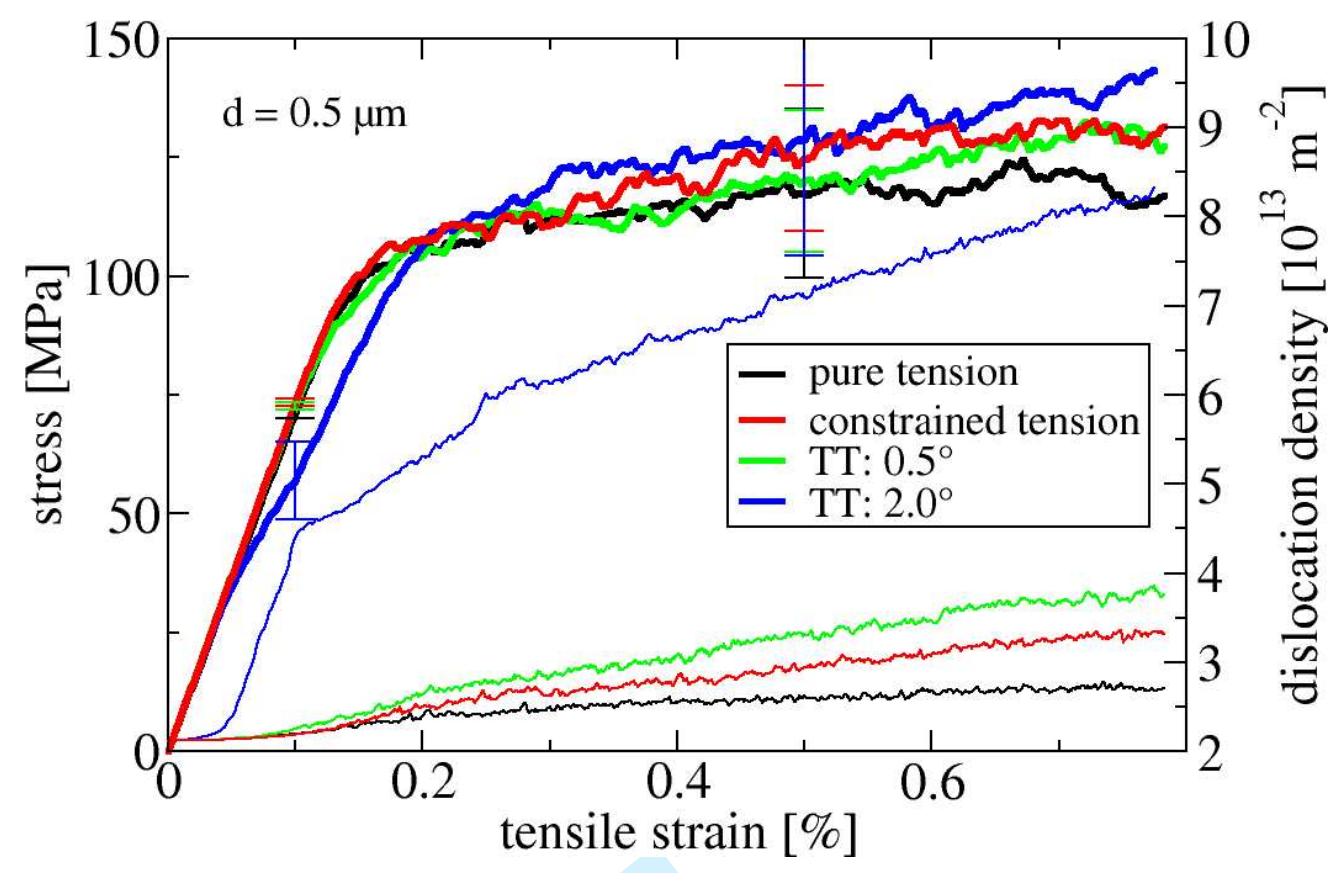




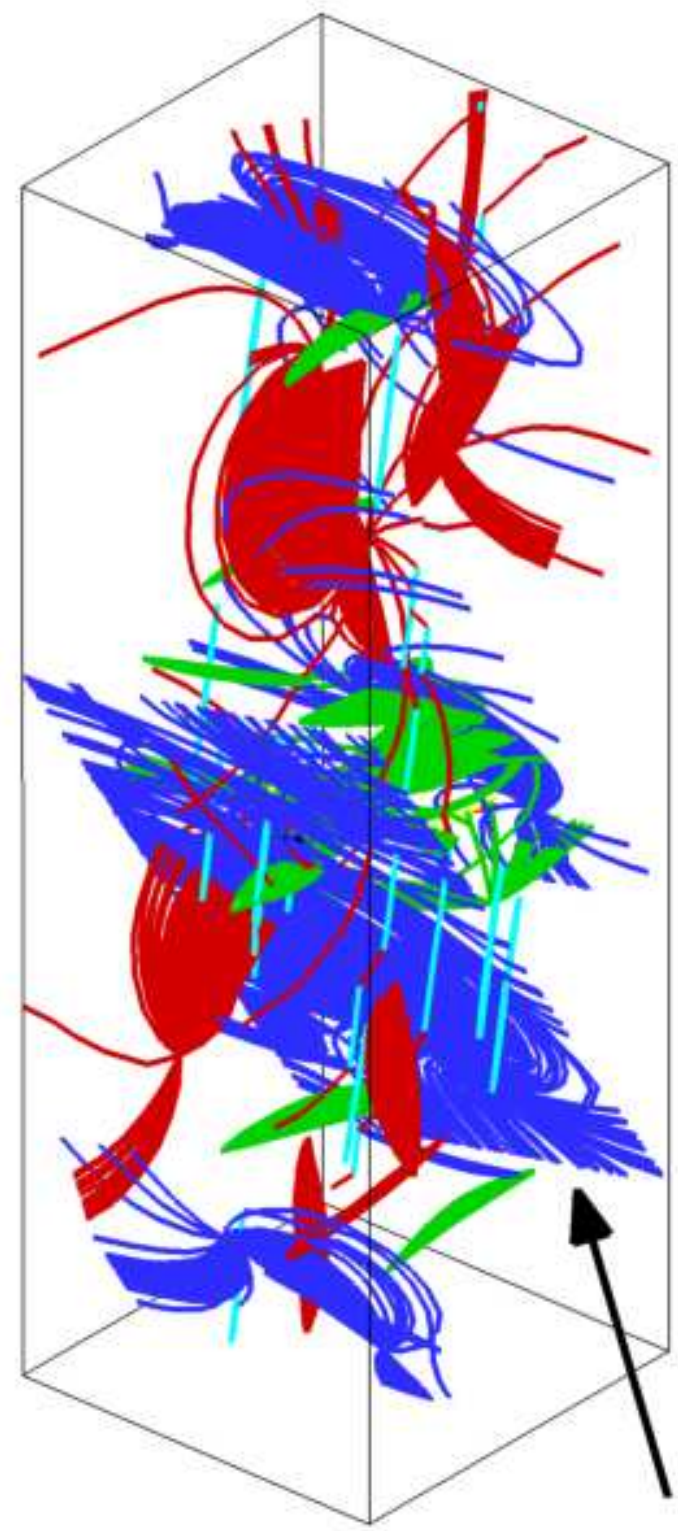

http://mc.manuscriptcentral.com/pm-pml 


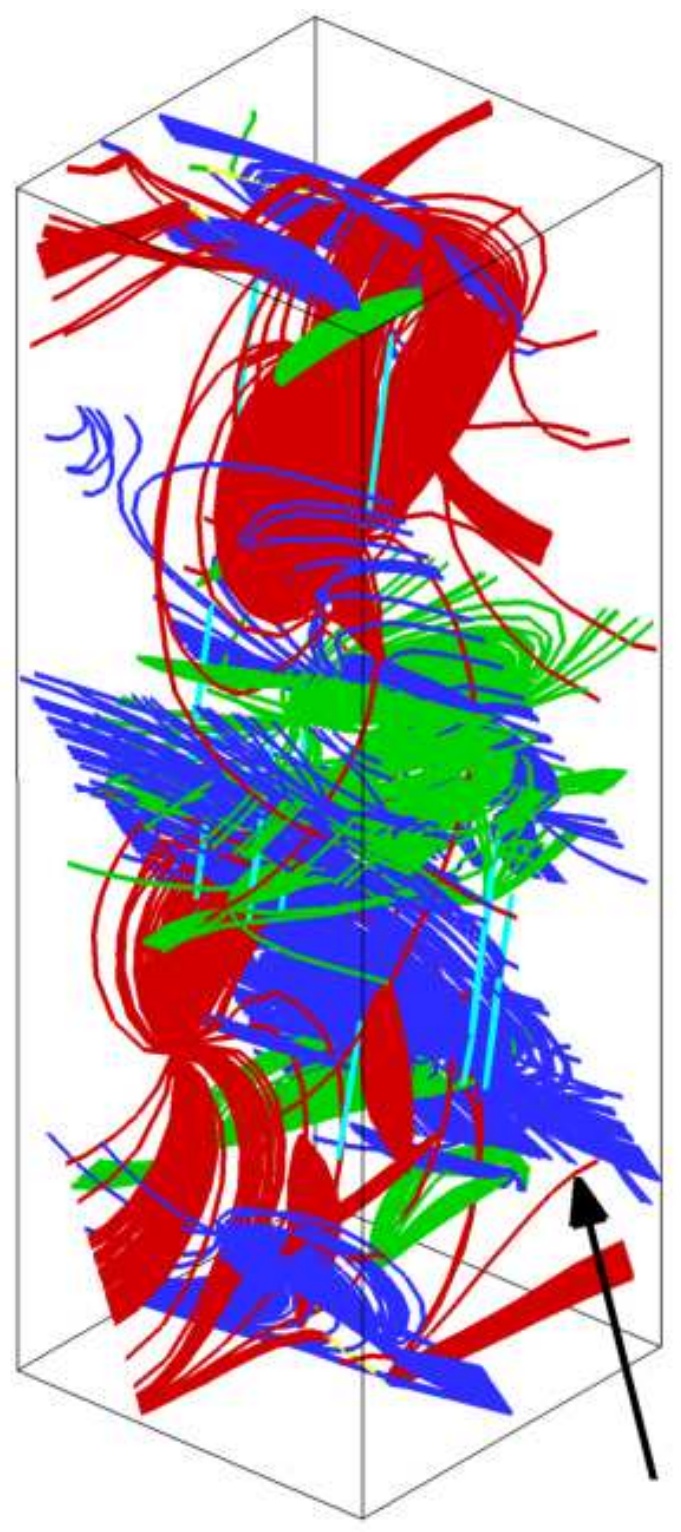




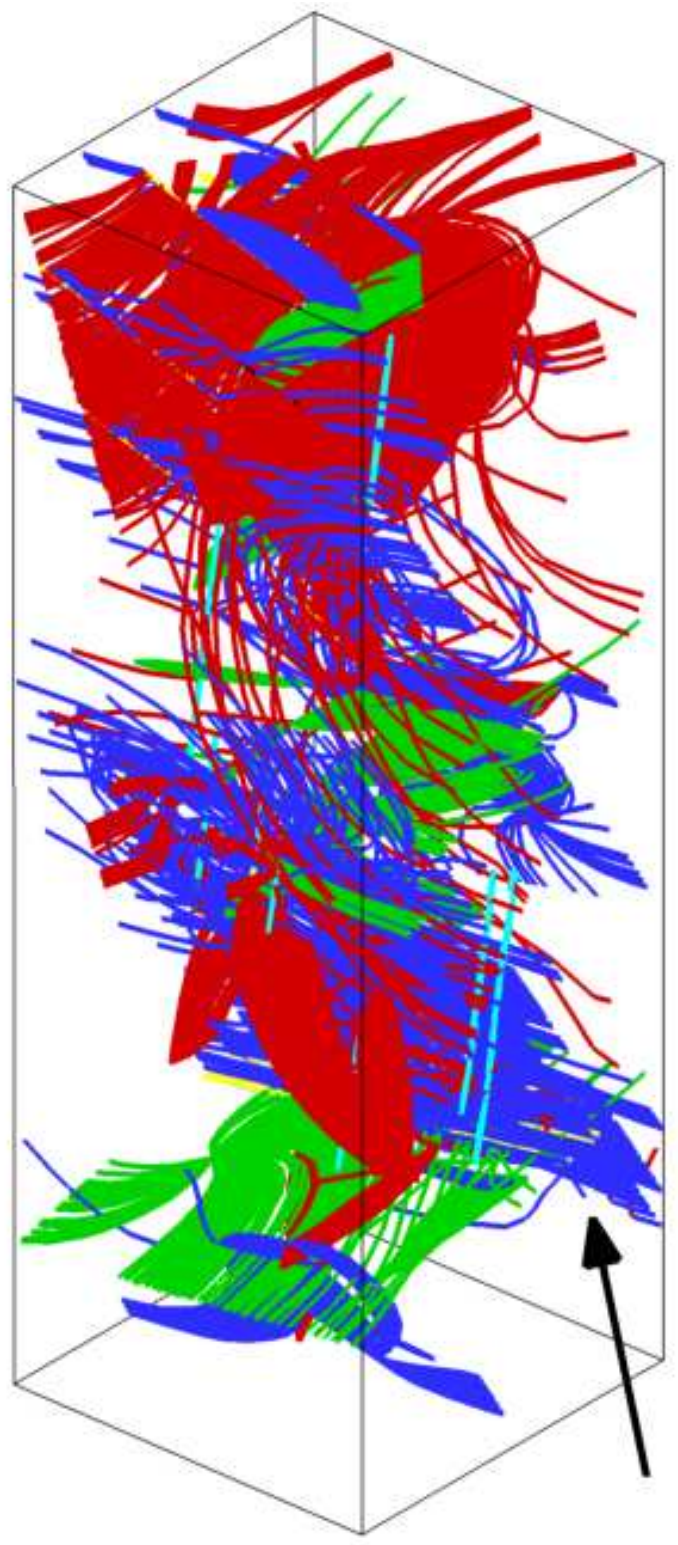




1
2
3
4
5
6
7
8
9
10
11
12
13
14
15
16
17
18
19
20
21
22
23
24
25
26
27
28
29
30
31
32
33
34
35
36
37
38
39
40
41
42
43
44
45
46
47
48
49
50
51
52
53
54
55
56
57
58
60

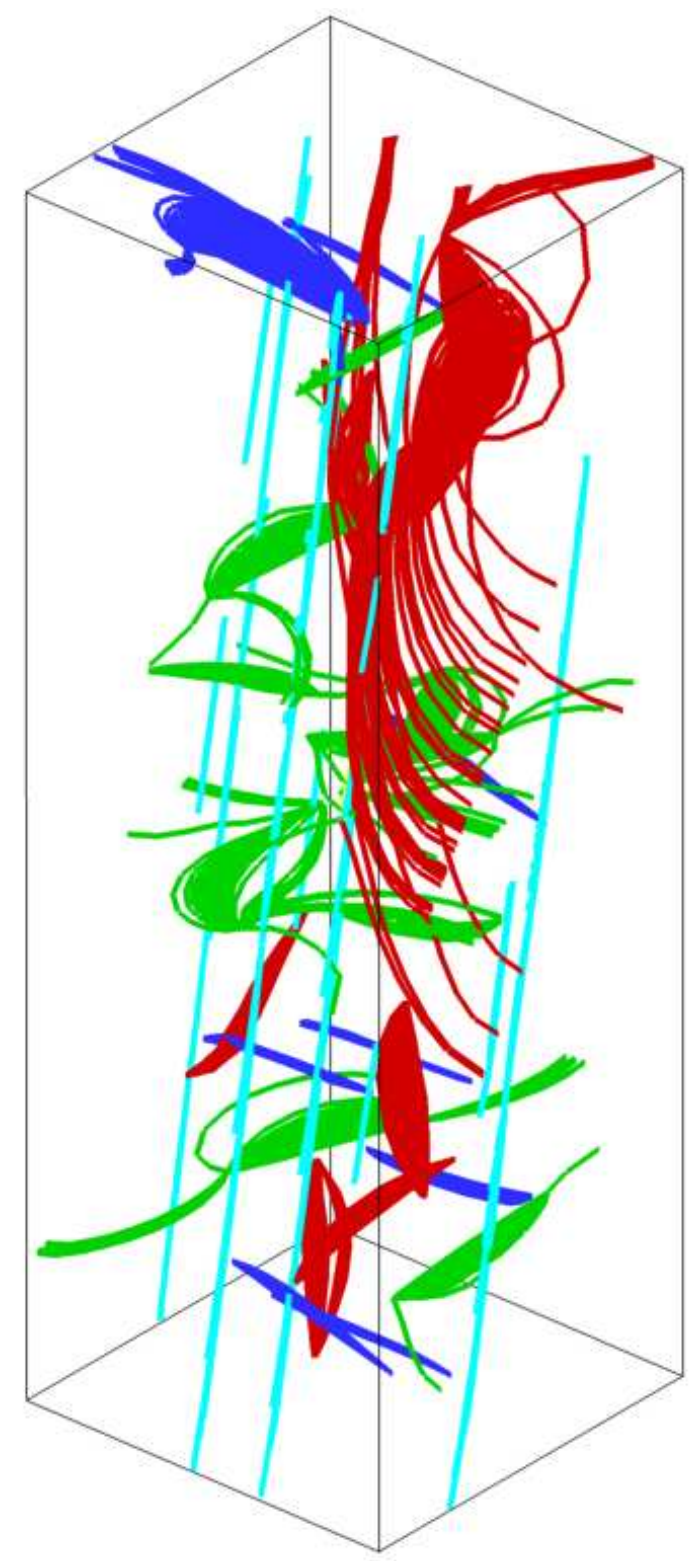

http://mc.manuscriptcentral.com/pm-pml 


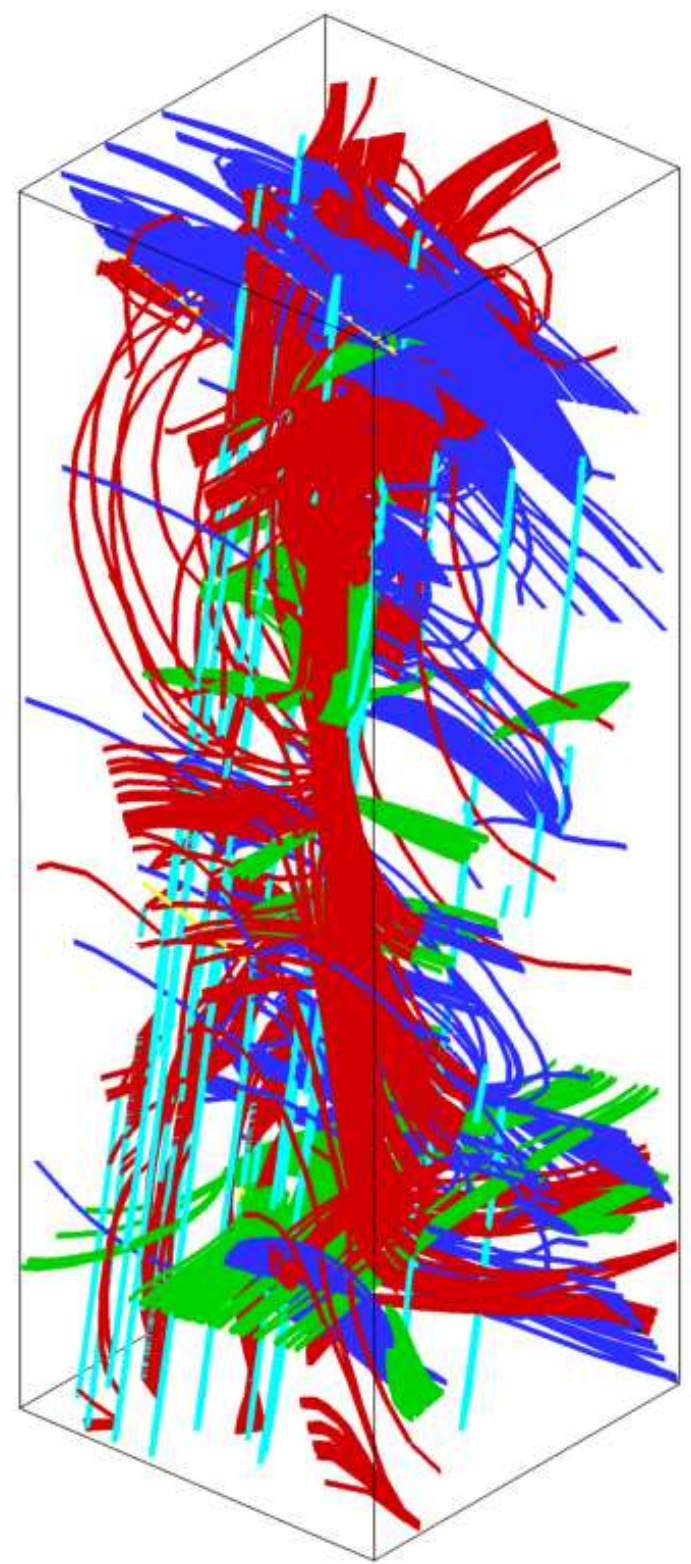




1
2
3
4
5
6
7
8
9
10
11
12
13
14
15
16
17
18
19
20
21
22
23
24
25
26
27
28
29
30
31
32
33
34
35
36
37
38
39
40
41
42
43
44
45
46
47
48
49
50
51
52
53
54
55
56
57
58
60

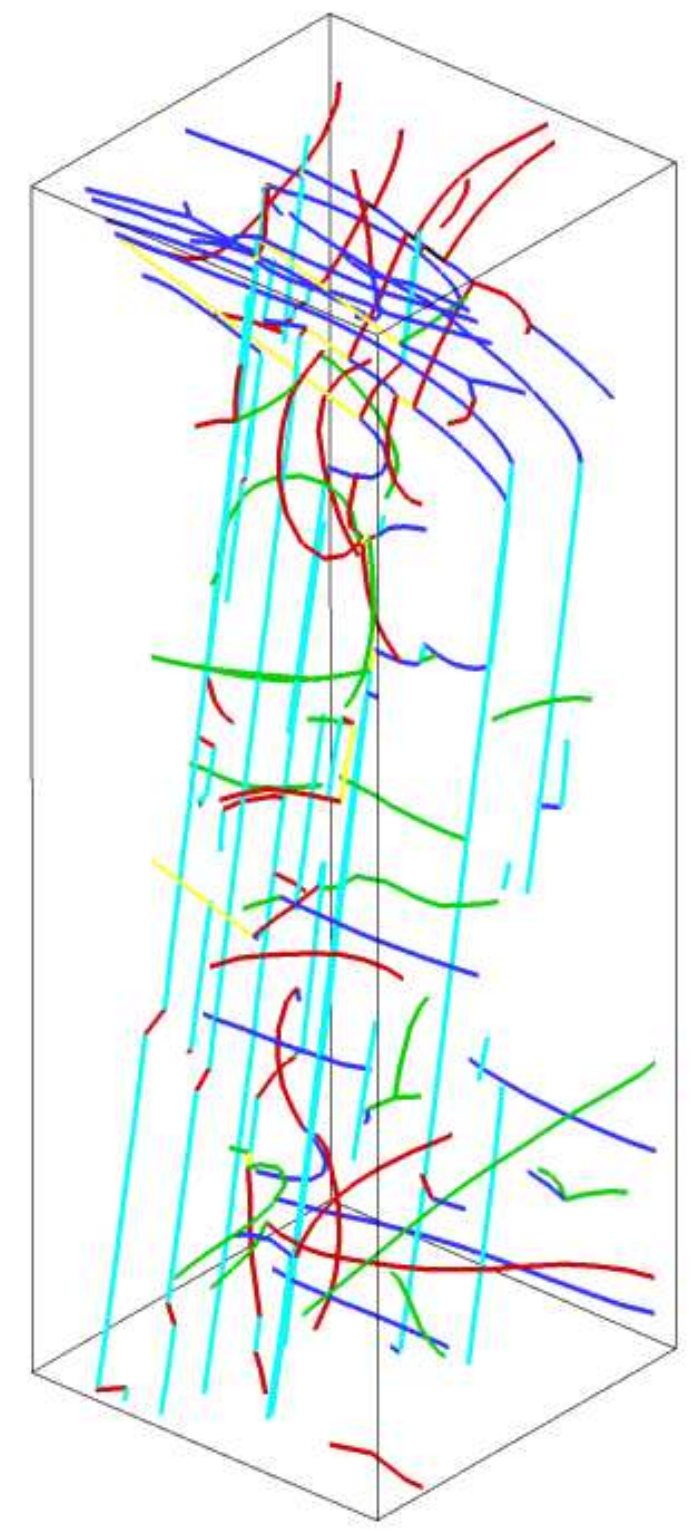

$128 \times 247 \mathrm{~mm}(72 \times 72 \mathrm{DPI})$

http://mc.manuscriptcentral.com/pm-pml 


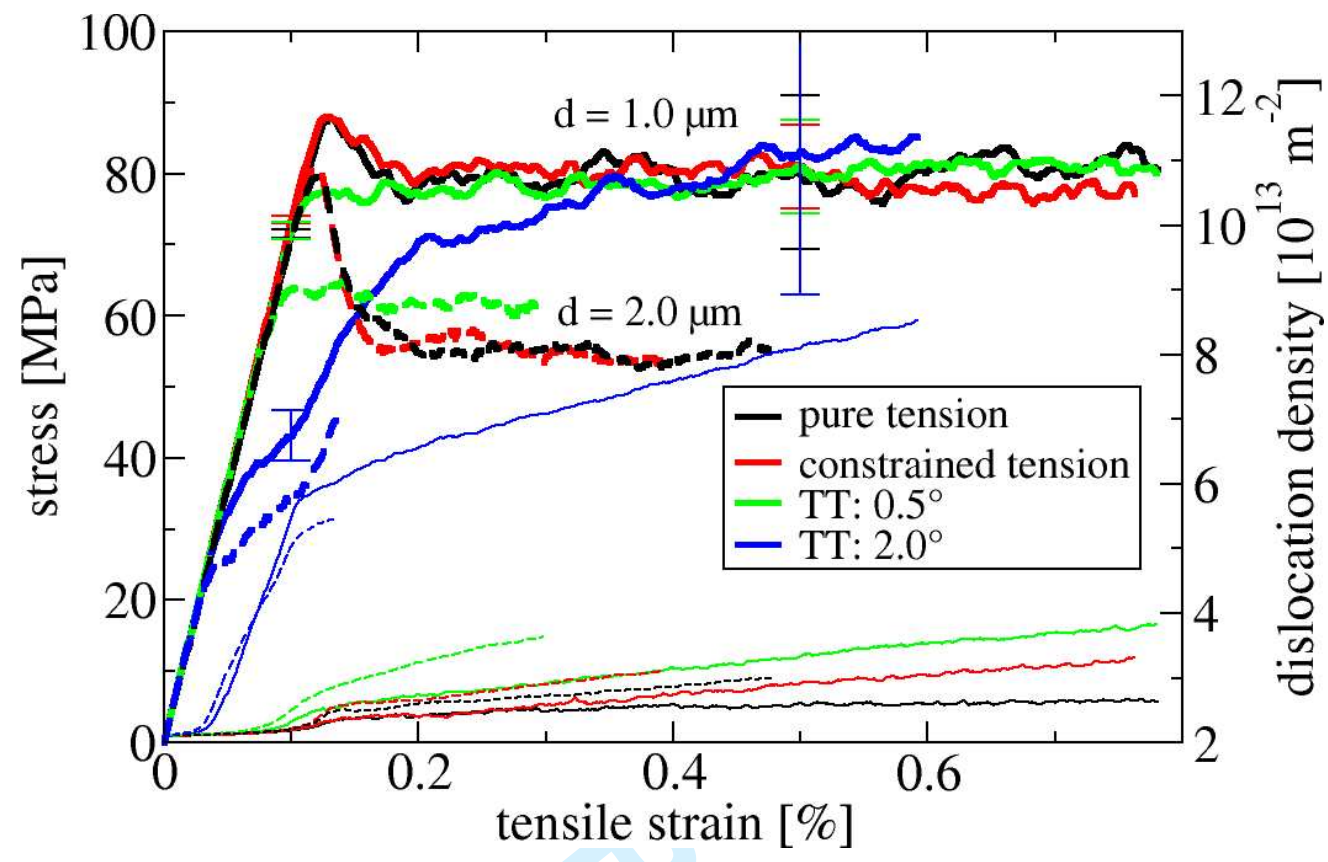




1
2
3
4
5
6
7
8
9
10
11
12
13
14
15
16
17
18
19
20
21
22
23
24
25
26
27
28
29
30
31
32
33
34
35
36
37
38
39
40
59
50
50
49
50
51
52
53
44
54

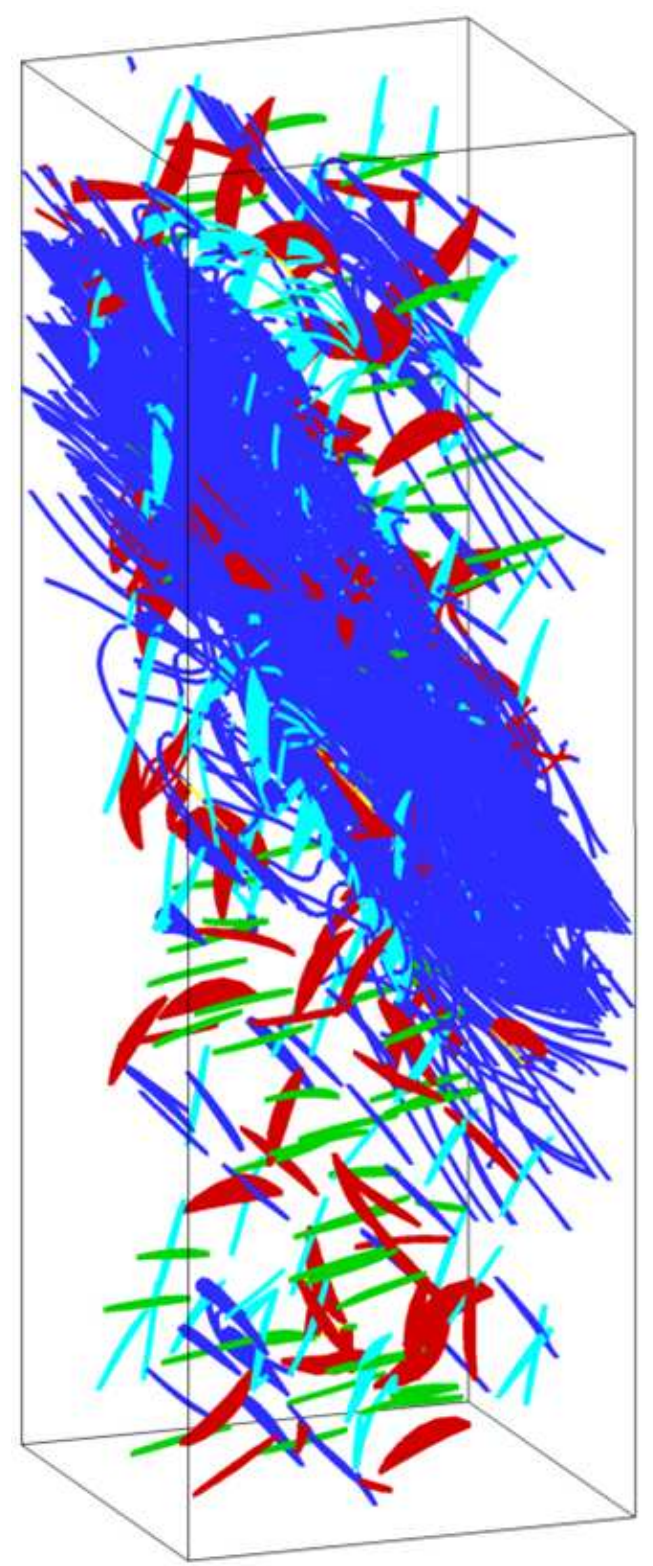




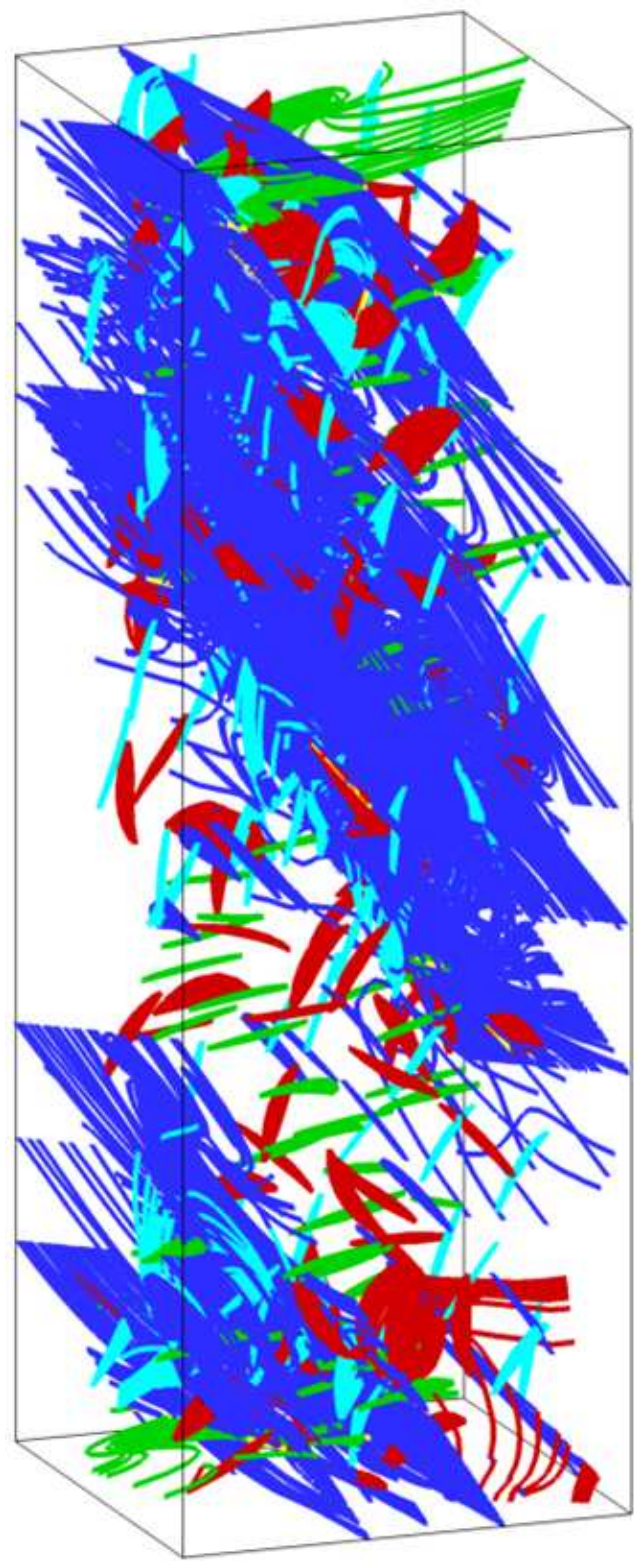




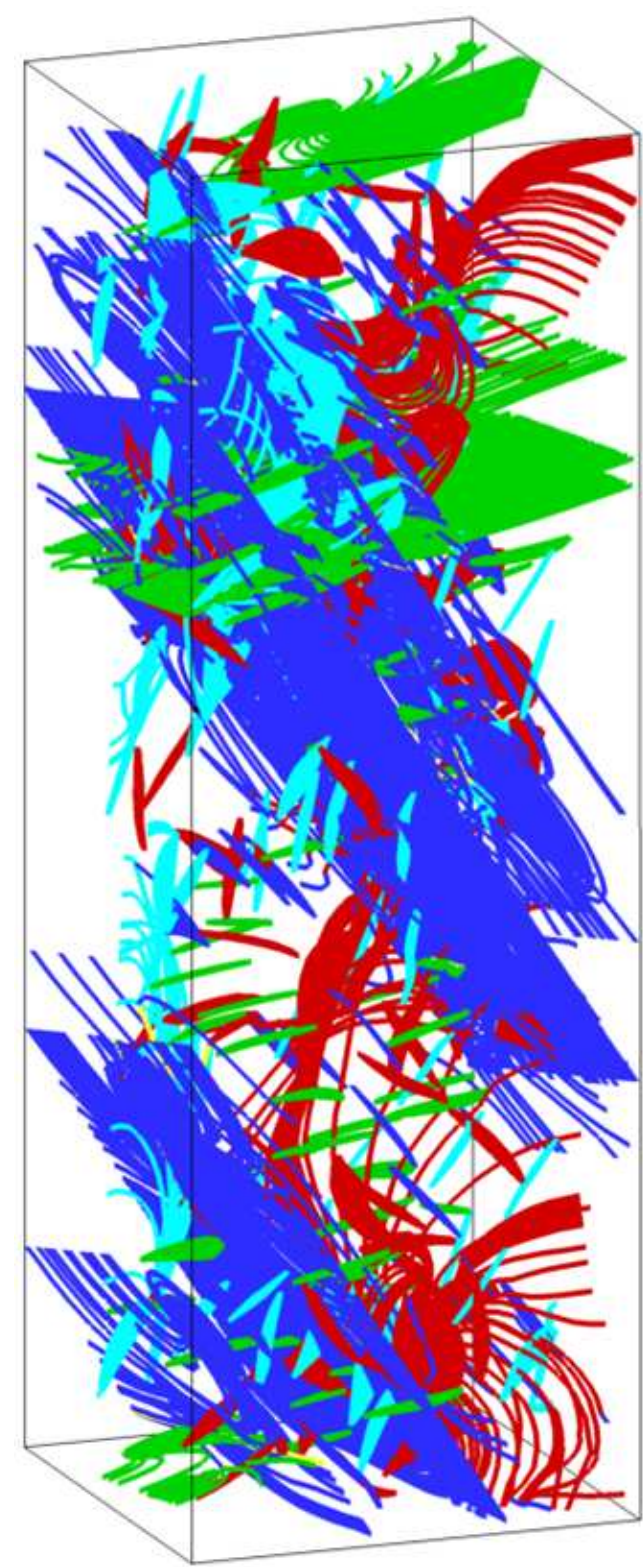

http://mc.manuscriptcentral.com/pm-pml 


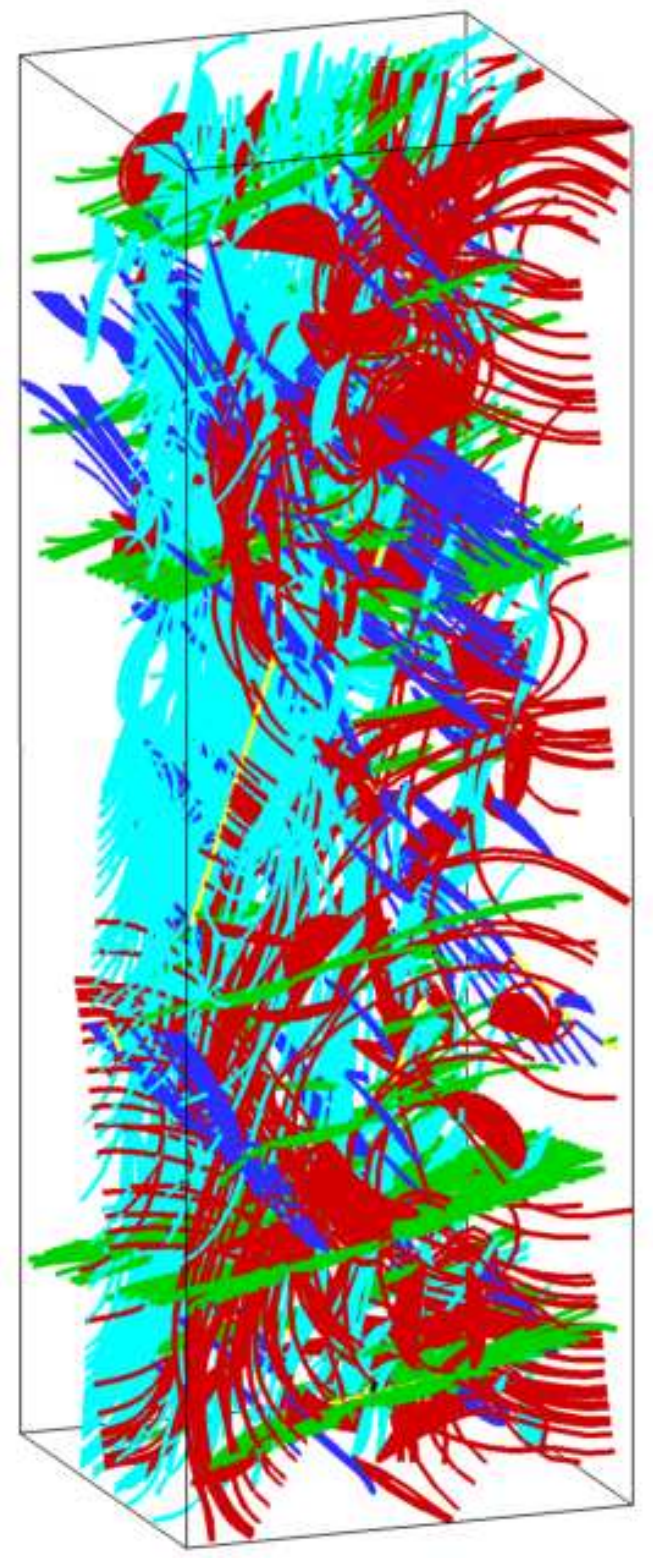

http://mc.manuscriptcentral.com/pm-pml 


1
2
3
4
5
6
7
8
9
10
11
12
13
14
15
16
17
18
19
20
21
22
23
24
25
26
27
28
29
30
31
32
33
34
35
36
37
38
39
40
41
42
43
44
45
46
47
50
58
59
50
52
53
50

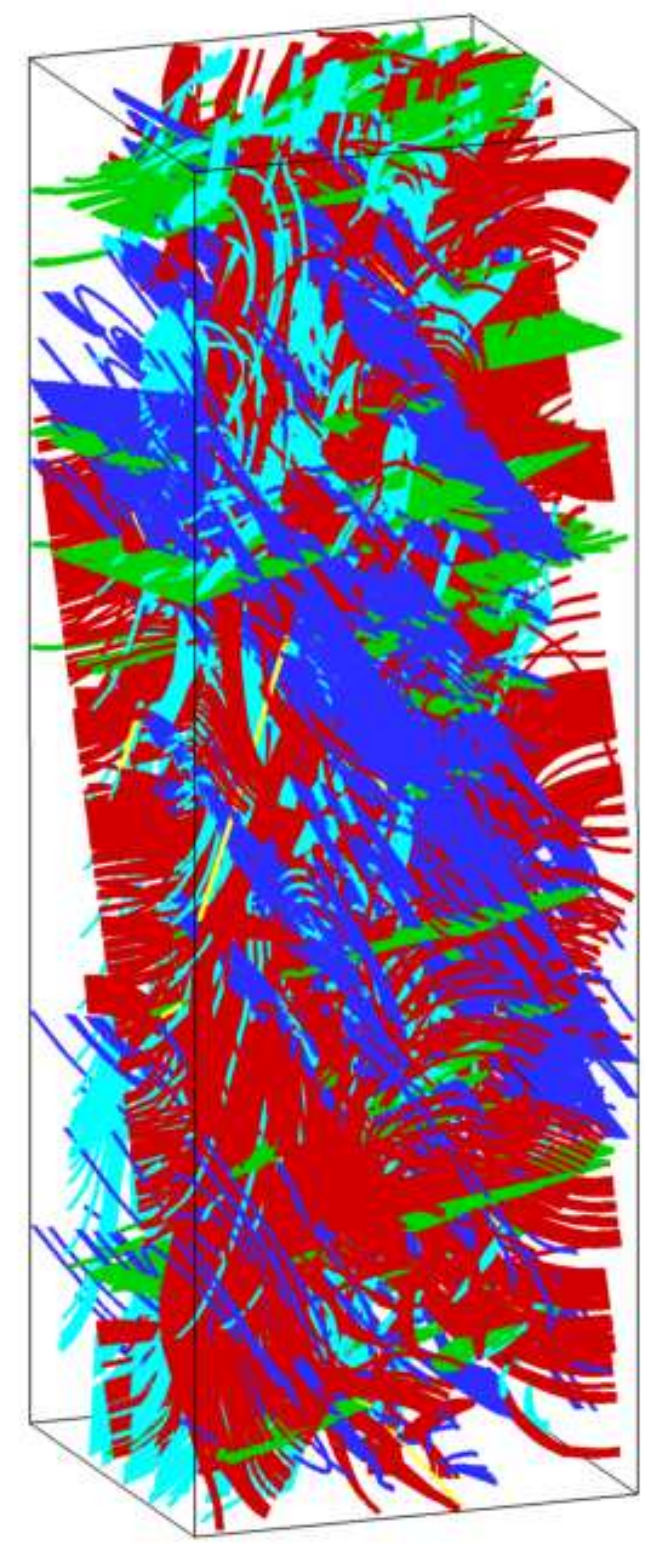




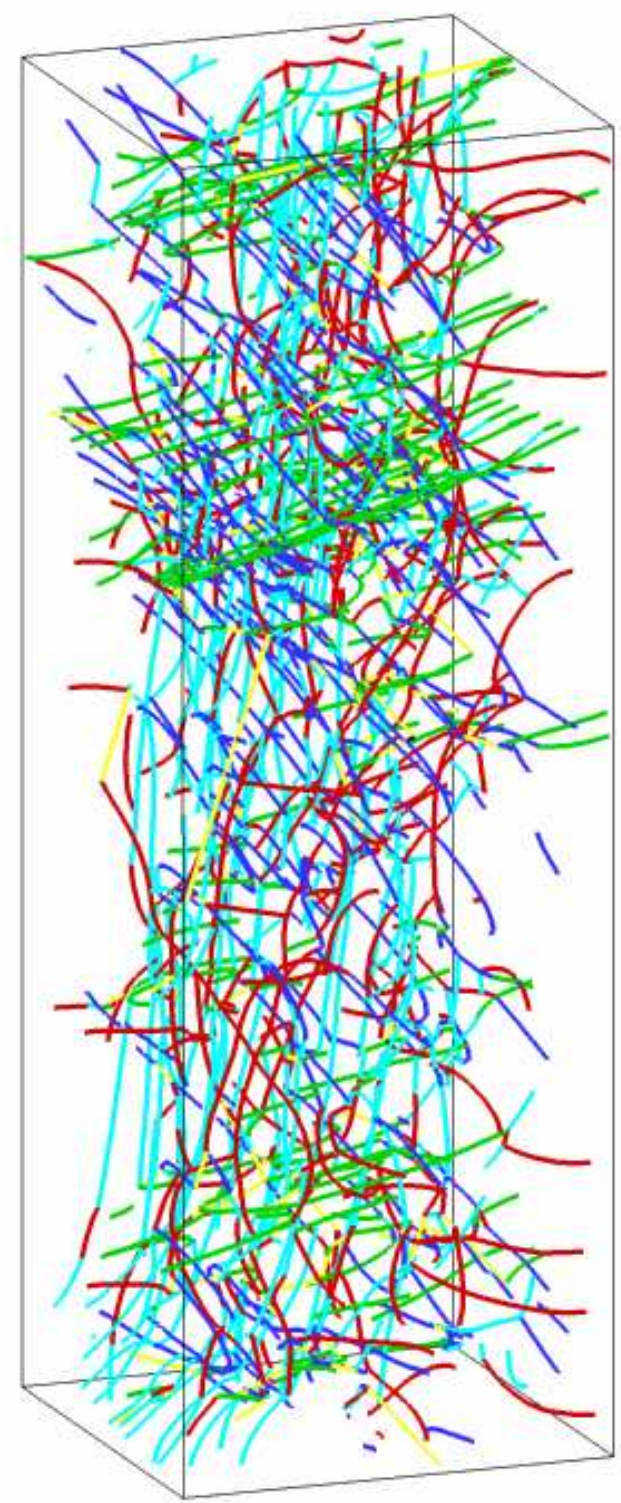

$125 \times 247 \mathrm{~mm}(72 \times 72 \mathrm{DPI})$

http://mc.manuscriptcentral.com/pm-pml 


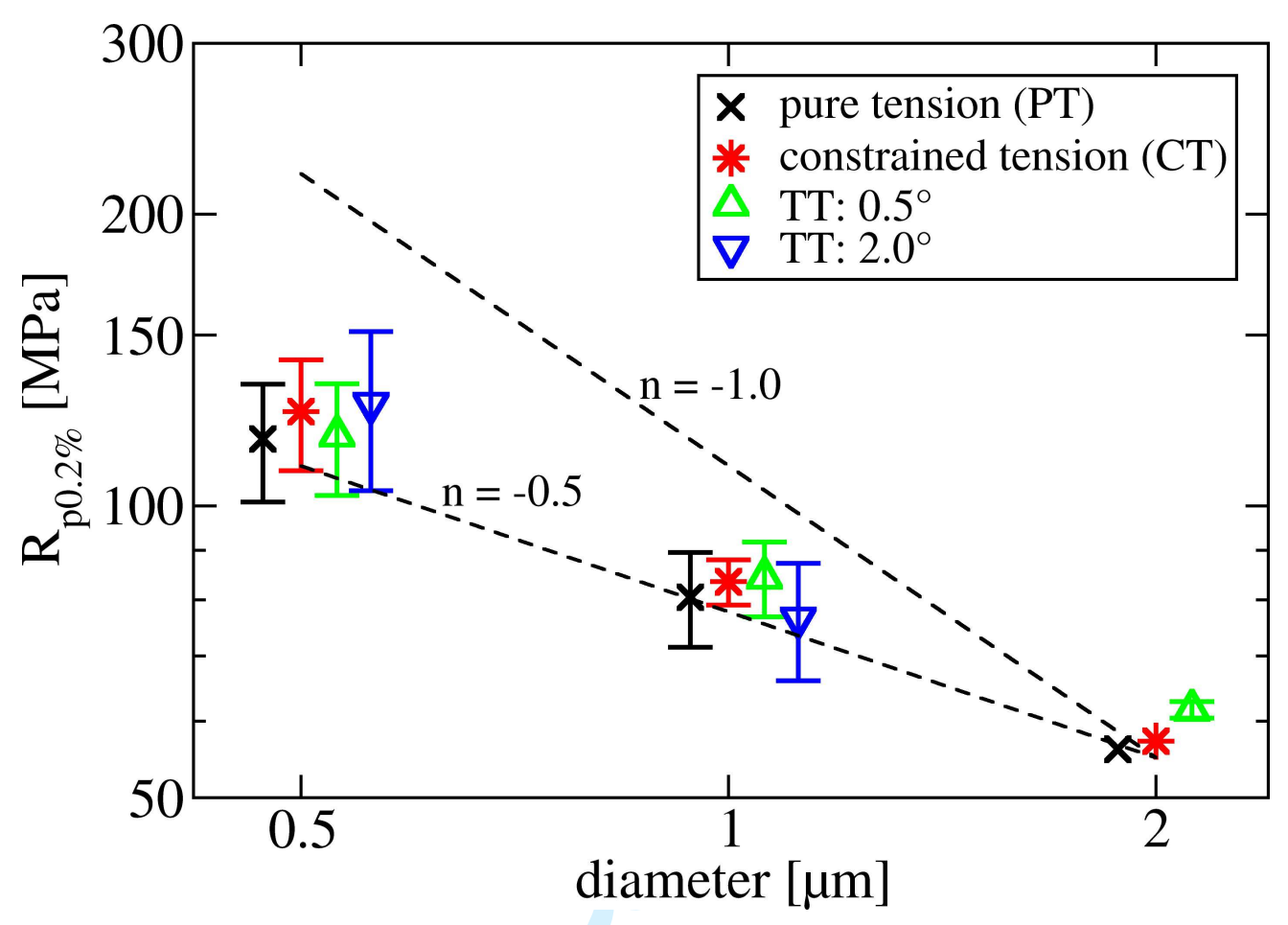

http://mc.manuscriptcentral.com/pm-pml 